\title{
Determining a Relation between X-ray Luminosity and Orbital Period of X-ray Binaries
}

\author{
Tyler Naffin \\ Department of Physics, University of Alberta
}

\begin{abstract}
The goal of this project was to examine the relationship between the average $x$-ray luminosity and the orbital period of $x$-ray binaries. Using the data gathered by the All-Sky Monitor instrument aboard the Rossi X-ray Timing Explorer, 29 sources were selected for investigation based on the intensity of the x-rays emitted from each of the sources. A literature search was then performed to gather further details on each of the sources, including orbital period, distance, hydrogen column density, and classification of each source as either low- or high-mass x-ray binaries with either neutron star or black hole companions. Sufficient data for 22 of the sources was known in order to create plots of the average luminosity versus the period, which allowed for the period-luminosity relationships for $\mathrm{x}$-ray binaries to be further examined.
\end{abstract}

\section{INTRODUCTION}

X-ray binaries (XRBs) are star systems that contain two gravitationally bound objects, one being a compact body; usually a neutron star or black hole, and the other a donor star. The donor star, being in close proximity to the compact body, begins to overflow its Roche lobe and matter accretes onto the compact body. Roche lobes are defined as the boundary between the two stars where the gravitational force is at an equipotential. The highly energetic nature of the mass transfer results in the emission of x-rays from the system. XRBs can be classified as either low-mass or high-mass $x$-ray binaries depending on the mass of the donor star[1].

Low-mass x-ray binaries (LMXBs) are defined by the mass of the donor star which typically has a mass of approximately two solar masses or less. They are typically characterized by the stars having a small orbital radii and period, with the period ranging anywhere from 11.4 minutes for the shortest known period, to 33.5 days for the longest known period, although longer periods arguably exist. The small separation between the two stars is required to facilitate the mass transfer due to the low mass of the system[2].

High-mass x-ray binaries (HMXBs) have a donor star with a mass that typically exceeds ten solar masses. As a result of the larger mass the separation of the two stars can be greater and consequently the orbital period can be longer, ranging from approximately 5 hours to 580 days in known cases. In the case of HMXBs, mass transfer can also be facilitated by strong solar winds, either in place of or in addition to the overflow of the Roche lobes[3].

XRBs that have donor stars with masses between two and ten solar masses, also known as intermediate-mass $\mathrm{x}$-ray binaries (IMXBs), are less common as the donor stars of IMXBs tend to quickly transfer much of their mass onto the compact body, and mimic the properties of LMXBs[4].

XRBs are thought to be created by one of two methods. In some cases, one of the stars in a binary star system can undergo a core-collapse supernova, and thus be transformed into either a neutron star or black hole. However the supernova can result in the binary system becoming gravitationally unbound, depending on how much mass is ejected. It is believed that at least half of the total mass of the binary system must be ejected for the system to become gravitationally unbound. Therefore systems with a much more massive companion star are more likely to remain gravitationally bound. The second method involves a compact body and another star becoming gravitationally bound after chance close encounter. This is more likely to occur in areas where the density of stars is much greater, such as globular clusters or the galactic core, which is consistent with observations where relatively high numbers of XRBs are observed[5].

One method for increasing our understanding of XRBs is to create models based on theory and observation such as luminosity functions. Luminosity functions are defined by the number of sources within a given luminosity interval. They can be used compare and confirm theoretical and observational results. In the case of x-rays, XRBs are a major source of $\mathrm{x}$-ray emissions in a standard galaxy, and therefore $\mathrm{x}$-ray luminosity functions can place constraints on the theoretical models of XRBs[6]. Another method for understanding the physical workings of XRBs is to compare how the x-ray luminosity of XRBs relates to the orbital period of the binary system. The process of mass transferring from the donor star to the compact object in an XRB can change both the luminosity and the period, and it was the goal of this project to analyze several XRBs and their luminosity and period and to see how they relate. The relation would hopefully provide insight into the general nature and evolution of XRBs, be they LMXBs or HMXBs.

\section{DATA ACQUISITION AND ANALYSIS}

The data used for this project was obtained by the All-Sky Monitor (ASM) aboard the Rossi X-ray Timing Explorer (RXTE) satellite. The RXTE contains three different instruments dedicated to detecting extra-solar $\mathrm{x}$ - 
rays, the Proportional Counter Array (PCA), the High Energy X-ray Timing Experiment (HEXTE), and the AllSky Monitor[7]. The ASM was capable of scanning 80\% of the sky for every 90 minute orbit of the earth, making it useful for gathering data from a large number of sources[8]. The RXTE was launched on December 30, 1995 and remained operational until it was decommissioned on January 5, 2012[9]. Late in the operational lifespan of the RXTE mission, the ASM instrument began to suffer from some technical faults. This was most clearly noticeable in the data from the Crab nebula where the typically constant x-ray flux dipped for a period of approximately 200 days by about 12 counts/s, down from its usual 75 counts/s. It should be noted that nothing was done to attempt to correct for this in the analysis.

The data provided by NASA's Godard Space Flight Center Guest Observer Facility offered the observations made of approximately 350 sources over the course of sixteen years, from January 1, 1996 until January 1, 2012, presenting the $x$-ray intensity, or count rate, for each source from 90 second dwells in a FITS format. This data has been further compiled and analyzed by a team at MIT, which offered the data in ASCII format, as well as providing plots of the data for the 2011 year for visual reference. In addition to providing the dwell-by-dwell data, the MIT team has provided one-day averages of the count rates for each of the sources.

The visual plots of the 2011 data were invaluable in selecting sources for analysis. By reviewing the plots of the data, any source that appeared to have ten or more "three-sigma" detections was noted down for further analysis. The "three-sigma" detections are the one-day averaged data points with smaller estimated uncertainties, or certainties to within three-sigma. The reasoning for this was the thought that the sources with numerous three-sigma detections would have the high or variable luminosities that were desired for further analysis.

A total of sixty-three data sources were selected from the initial review, plus an additional eight sources were selected after a brief literature search for other possible strong sources. It is however possible that some strong sources may have been passed over if the 2011 data was unremarkable, and if the literature search turned up no results.

For each of the seventy-one sources, the ASCII data for the one-day averages were copied from the MIT website, which included the date of the observation in Modified Julian Days (MJD), the count rate, and the count rate error. The ASCII files were run through a Perl script to obtain five-day averages of the count rate in an attempt to further simplify the data. From here the data was plotted using the graphing software OriginPro 8 to obtain a visual reference for all the data from each source.

A somewhat arbitrary selection process was used from this point to determine which sources were viable candidates for continued analysis. Numerous

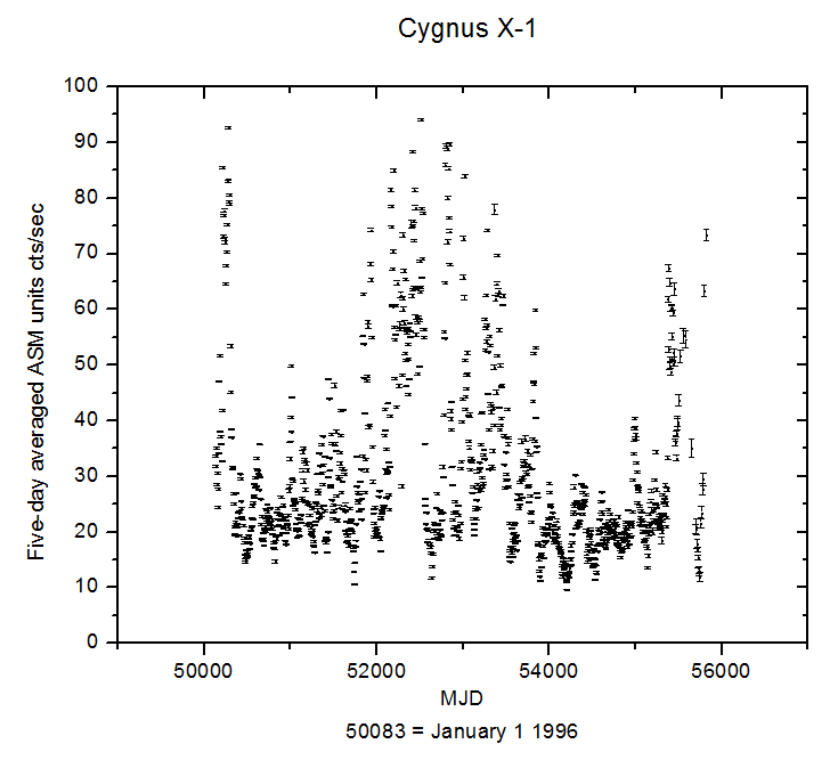

FIG. 1. Plot of the five-day averaged ASCII data showing the $\mathrm{x}$-ray intensity in counts/s from the source Cyg X-1 from January 1, 1996 to January 1, 2012.

sources were rejected for their average count rates were rather low, not exceeding 10 to 15 counts per second. There were twenty-nine sources that had proportionately higher average count rates, or in some cases if the source experienced a strong, short burst of x-rays, and were selected for further analysis. In figure 1 , the plot of the count rate for Cyg X-1 is shown as an example.

Once the twenty-nine sources had been selected, a literature search was then begun in order to classify the XRBs as either HMXBs or LMXBs, and whether the companion star was a neutron star or black hole. Additional facts about each source that were required were the orbital period of the $\mathrm{XRB}$, the distance of the system, and the hydrogen column density $\left(\mathrm{N}_{H}\right)$, all of which are listed on table 1.

Most of the values were gathered from various catalogs, although there were notable exceptions. While most distances were known, for some it was required to estimate a distance, for which an estimate of $8 \mathrm{kpc}$ was used. Each of the sources with an unknown distance had the prefix GX, which indicates the source likely lies near the galactic core, at a distance of approximately $8 \mathrm{kpc}$. For some sources the known distance was given as a range of values. In these cases the average between the two extremes was used as the distance. For the cases in which the hydrogen column density was unknown, the relation $\mathrm{N}_{H}=5.3 \times 10^{21} \mathrm{~cm}^{-2} \mathrm{E}_{B-V}$ found in Predehl et. al. 1994 was used, where the values for interstellar reddening $\left(\mathrm{E}_{B-V}\right)$ were readily found in the literature, and thus enabling $\mathrm{NH}$ to be found.

Once the values for the hydrogen column density were known, they were used in conjunction with the average count rate, which had been determined by 
taking the weighted average of the count rate for all data points, to determine the average $x$-ray flux of each source. Using the Portable, Interactive MultiMission Simulator (PIMMS v4.4) made available on-line by the Chandra X-ray Center, the count rate was converted to the unabsorbed flux of the source in units of ergs $/ \mathrm{cm}^{2} / \mathrm{s}$. The PIMMS program is designed to enable the conversion of flux and count rates from various missions, including the RXTE ASM data. In its flux calculations for each source PIMMS requires the hydrogen column density, and the photon index, a necessary parameter for power law calculations where intensity is proportional to the energy of the photons to the negative power of the photon index. For all calculations, a photon index of 2 was used, which corresponds to the inverse square law.

Armed with the x-ray flux for each source, the luminosity could easily be determined by multiplying the flux by $4 \pi$ times the distance in centimetres, giving the $\mathrm{x}$-ray luminosity in ergs/s. Finally plots of the luminosity versus period could be created from the data. For seven of the twenty-nine source the orbital periods were unknown, and were therefore not included in the plots. Three plots were created, one plot displaying the neutron star LMXBs, another with the black hole LMXBs, and the final with all the HMXBs together.

\section{RESULTS}

The plots of the data yielded some interesting results. In the plot of the LMXBs with neutron star companions (figure 2.1), which contained twelve of the twenty-nine sources, the data did not behave entirely as expected. In Podsiadlowski et. al. 2001 an analysis done on LMXBs and IMXBs figures that the luminosity increases as the orbital period increases, although it should be noted that in the paper the mass transfer rate is used rather than the luminosity, which is proportional by the relation $10^{-8} \mathrm{Msun} / \mathrm{yr}=10^{38} \mathrm{ergs} / \mathrm{s}$. The mass transfer rate is thought to be proportional to the luminosity by the fact that the $x$-rays emitted by an XRB are caused by the highly energetic in falling mass from the donor star to the compact object, with a greater mass transfer rate resulting in more $x$-rays being emitted, and therefore a proportional relation exists. Figure 3, taken from the Podsiadlowski paper, illustrates the relation. In contrast the results from the ASM data indicates that for periods on the order of 20 hours or less the luminosity varies from $2.44 \times 10^{36} \mathrm{ergs} / \mathrm{s}$ in the case of Aql X-1, to $5.82 \times 10^{38} \mathrm{ergs} / \mathrm{s}$ in the case of Sco X-1, both of which have very similar periods. However for longer periods on the order of 100s of hours all the sources display consistently higher luminosities.

The plot of the LMXBs with black hole companions (figure 2.2), of which there were five sources, returned more predictable results, where the shorter orbital periods had lower luminosities, and longer periods had

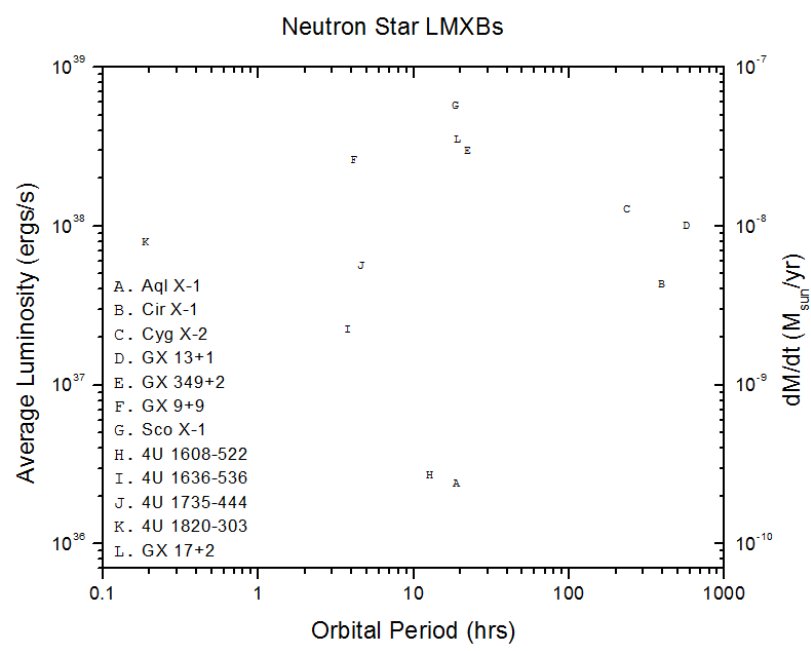

(1) LMXBs with neutron star companions.

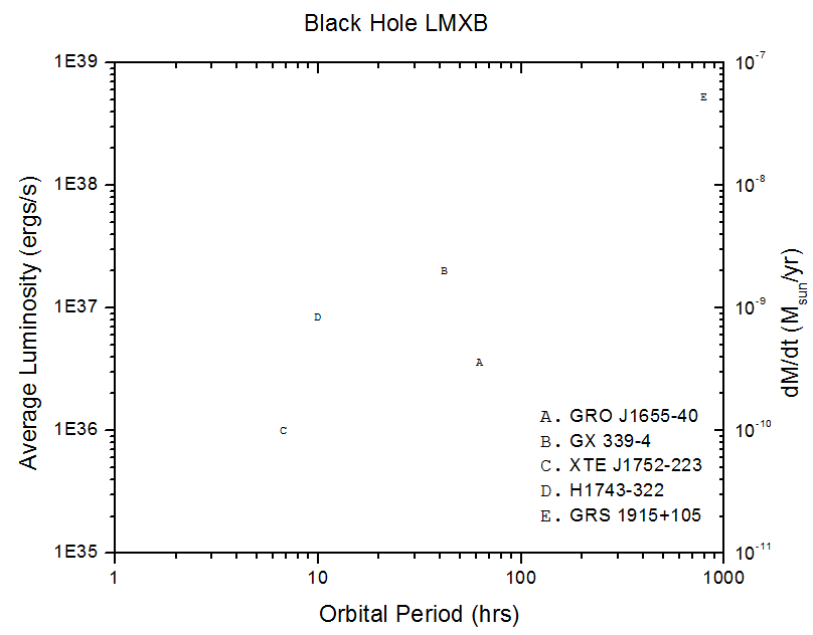

(2) LMXBs with black hole companions.

HMXB

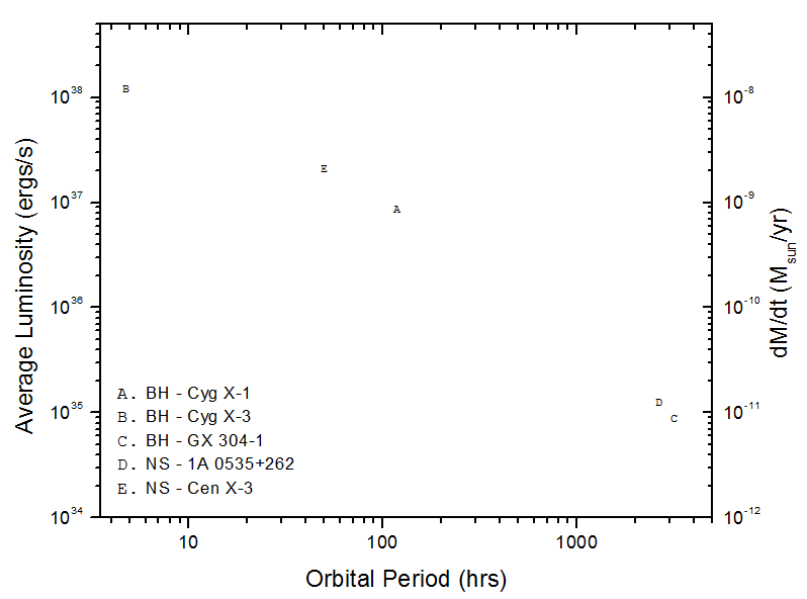

(3) HMXBs with both neutron star and black hole companions. The neutron star and black hole companions are distinguished by the NS and $\mathrm{BH}$ labels on the plot, respectively.

FIG. 2. The average x-ray luminosity and the mass transfer rate are plotted together on the $y$-axis. 


\begin{tabular}{|c|c|c|c|c|c|}
\hline Source & Period & Distance & Classification & $\begin{array}{l}\text { Hydrogen Column } \\
\text { Density } \mathbf{N}_{H}\left(\mathrm{~cm}^{-2}\right)\end{array}$ & $\begin{array}{l}\text { Luminosity } \\
\text { (ergs/s) }\end{array}$ \\
\hline Aql X-1 & $\begin{array}{c}18.95 \mathrm{~h} \\
\text { (Liu 2008) }\end{array}$ & $\begin{array}{c}5 \mathrm{kpc} \\
\text { (Liu 2008) }\end{array}$ & NS, LMXB & $\begin{array}{c}3.60 \mathrm{E}+21 \\
\text { (Bodaghee 2008) }\end{array}$ & $2.44 \mathrm{E}+36$ \\
\hline Cir X-1 & $\begin{array}{c}398.4 h \\
(\text { Liu 2008) }\end{array}$ & $\begin{array}{c}5.5 \mathrm{kpc} \\
(4-12 \mathrm{kpc}) \\
(\text { Liu 2008) }\end{array}$ & NS, LMXB & $\begin{array}{c}1.60 \mathrm{E}+22 \\
\text { (Bodaghee 2008) }\end{array}$ & $4.36 \mathrm{E}+37$ \\
\hline Cyg X-2 & $\begin{array}{c}236.2 \mathrm{~h} \\
(\mathrm{Liu} 2008)\end{array}$ & $\begin{array}{c}7.2 \mathrm{kpc} \\
(\text { Liu 2008) }\end{array}$ & NS, LMXB & $\begin{array}{c}1.90 \mathrm{E}+21 \\
\text { (Bodaghee 2008) }\end{array}$ & $1.30 \mathrm{E}+38$ \\
\hline GX 13+1 & $\begin{array}{c}577.6 h \\
(\operatorname{Liu} 2008)\end{array}$ & $\begin{array}{c}71 \mathrm{kpc} \\
\text { (Liu 2008) }\end{array}$ & NS, LMXB & $\begin{array}{c}3.20 \mathrm{E}+22 \\
\text { (Bodaghee 2008) }\end{array}$ & $1.01 \mathrm{E}+38$ \\
\hline GX 349+2 & $\begin{array}{l}\text { 22.5h (14.9d?) } \\
\quad(\text { Liu 2008) }\end{array}$ & $\begin{array}{c}9.2 \mathrm{kpc} \\
(\mathrm{Liu} 2008)\end{array}$ & NS, LMXB & $\begin{array}{c}\text { 6.73E+21 } \\
\text { (Bodaghee 2008) }\end{array}$ & $3.01 \mathrm{E}+38$ \\
\hline GX 9+9 & $\begin{array}{c}4.20 \mathrm{~h} \\
(\mathrm{Liu} 2008)\end{array}$ & $10 \mathrm{kpc}(?)$ & NS, LMXB & $\begin{array}{c}2.60 \mathrm{E}+21 \\
\text { (Predehl 1995) }\end{array}$ & $2.63 E+38$ \\
\hline Sco X-1 & $\begin{array}{c}\text { 18.90h } \\
\text { (Liu 2008) }\end{array}$ & $\begin{array}{c}2.8 \mathrm{kpc} \\
(\mathrm{Liu} 2008)\end{array}$ & NS, LMXB & $\begin{array}{c}\text { 2.37E+22 } \\
\text { (Bodaghee 2008) }\end{array}$ & $5.82 \mathrm{E}+38$ \\
\hline 4U 1608-522 & $\begin{array}{c}12.89 \mathrm{~h} \\
\text { (Liu 2008) }\end{array}$ & $\begin{array}{l}\text { 3.6kpc, } 4.1 \mathrm{kpc} \\
\quad(\mathrm{Liu} 2008)\end{array}$ & NS, LMXB & $\begin{array}{c}1.28 \mathrm{E}+22 \\
\text { (Bodaghee 2008) }\end{array}$ & $2.73 E+36$ \\
\hline 4U 1636-536 & $\begin{array}{c}3.80 \mathrm{~h} \\
\text { (Liu 2008) }\end{array}$ & $\begin{array}{c}6 \mathrm{kpc} \\
(\text { Liu 2008) }\end{array}$ & NS, LMXB & $\begin{array}{c}4.20 \mathrm{E}+21 \\
\text { (Bodaghee 2008) }\end{array}$ & $2.27 \mathrm{E}+37$ \\
\hline 4U 1735-444 & $\begin{array}{c}4.65 \mathrm{~h} \\
\text { (Liu 2008) }\end{array}$ & $\begin{array}{c}7-9 \mathrm{kpc} \\
(\text { Liu 2008) }\end{array}$ & NS, LMXB & $\begin{array}{c}3.40 \mathrm{E}+21 \\
\text { (Bodaghee 2008) }\end{array}$ & $5.67 \mathrm{E}+37$ \\
\hline 4U 1820-303 & $\begin{array}{c}\text { 0.19h } \\
\text { (Liu 2008) }\end{array}$ & $\begin{array}{c}7.6 \mathrm{kpc} \\
\text { (Liu 2008) }\end{array}$ & NS, LMXB & $\begin{array}{c}1.60 \mathrm{E}+21 \\
\text { (Bodaghee 2008) }\end{array}$ & $8.00 \mathrm{E}+37$ \\
\hline GX 17+2 & $\begin{array}{c}19.5 \mathrm{~h} \\
\text { (Hertz 1986) }\end{array}$ & $\begin{array}{c}9.8 \mathrm{kpc} \\
\text { (Liu 2008) }\end{array}$ & NS, LMXB & $\begin{array}{c}2.00 \mathrm{E}+22 \\
\text { (Bodaghee 2008) }\end{array}$ & $3.52 \mathrm{E}+38$ \\
\hline GX 3+1 & unknown & $\begin{array}{c}\text { 6.5kpc } \\
\text { (Liu 2008) }\end{array}$ & NS, LMXB & $\begin{array}{c}1.59 \mathrm{E}+22 \\
\text { (Bodaghee 2008) }\end{array}$ & $7.16 \mathrm{E}+37$ \\
\hline GX $340+0$ & unknown & $8 \mathrm{kpc}(?)$ & NS, LMXB & $\begin{array}{c}3.90 \mathrm{E}+22 \\
\text { (Bodaghee 2008) }\end{array}$ & $1.78 \mathrm{E}+38$ \\
\hline GX 5-1 & unknown & $8 \mathrm{kpc}(?)$ & NS, LMXB & $\begin{array}{c}2.20 \mathrm{E}+22 \\
\text { (Bodaghee 2008) }\end{array}$ & $3.73 E+38$ \\
\hline GX 9+1 & unknown & $\begin{array}{c}5 \mathrm{kpc} \\
\text { (Liu 2008) }\end{array}$ & NS, LMXB & $\begin{array}{c}8.00 \mathrm{E}+21 \\
\text { (Bodaghee 2008) }\end{array}$ & $3.45 \mathrm{E}+37$ \\
\hline Ser X-1 & unknown & $\begin{array}{c}8.4 \mathrm{kpc} \\
(\text { Liu 2008) }\end{array}$ & NS, LMXB & $\begin{array}{c}5.00 \mathrm{E}+21 \\
\text { (Bodaghee 2008) }\end{array}$ & $8.05 E+37$ \\
\hline 4U 1705-440 & unknown & $\begin{array}{c}7.4 \mathrm{kpc} \\
(\text { Liu 2008) }\end{array}$ & NS, LMXB & $\begin{array}{c}1.42 \mathrm{E}+22 \\
\text { (Bodaghee 2008) }\end{array}$ & $4.77 \mathrm{E}+37$ \\
\hline GRO J1655-40 & $\begin{array}{c}\text { 62.88h } \\
\text { (Liu 2008) }\end{array}$ & $\begin{array}{c}3.2 \mathrm{kpc} \\
\text { (but }<1.7 \mathrm{kpc}) \\
(\text { Liu 2008) }\end{array}$ & BH, LMXB & $\begin{array}{c}5.80 \mathrm{E}+21 \\
\text { (Bodaghee 2008) }\end{array}$ & $3.61 E+36$ \\
\hline GRS 1915+105 & $\begin{array}{c}33.5 d \\
(\text { Liu 2008) }\end{array}$ & $\begin{array}{l}11.2-12.5 \mathrm{kpc} \\
(\text { Liu 2008) }\end{array}$ & BH, LMXB & $\begin{array}{c}1.98 \mathrm{E}+22 \\
\text { (Bodaghee 2008) }\end{array}$ & $5.26 \mathrm{E}+38$ \\
\hline GX339-4 & $\begin{array}{c}42.14 \mathrm{~h} \\
(\text { Liu 2008) }\end{array}$ & $\begin{array}{l}>6 \mathrm{kpc}(8 \mathrm{kpc} ?) \\
\quad(\text { Liu 2008) }\end{array}$ & BH, LMXB & $\begin{array}{c}3.90 \mathrm{E}+21 \\
\text { (Bodaghee 2008) }\end{array}$ & $2.01 \mathrm{E}+37$ \\
\hline XTE J1752-223 & $\begin{array}{c}<6.8 \mathrm{~h} \\
\text { (Ratti 2012) }\end{array}$ & $\begin{array}{c}\text { 3.5-8kpc } \\
\text { (Ratti 2012) }\end{array}$ & BH, LMXB & $\begin{array}{c}5.00 \mathrm{E}+21 \\
\text { (Bodaghee 2008) }\end{array}$ & $1.00 \mathrm{E}+36$ \\
\hline H1743-322 & $\begin{array}{c}>10 \mathrm{~h} \\
\text { (Jonker 2009) }\end{array}$ & $\begin{array}{c}\text { 8.5kpc } \\
(\text { Liu 2008) }\end{array}$ & BH, LMXB & $\begin{array}{c}1.84 E+22 \\
\text { (Predehl 1995) }\end{array}$ & $8.44 \mathrm{E}+36$ \\
\hline Swift J1753.5-0127 & unknown & $\begin{array}{c}6 \mathrm{kpc} \\
(\mathrm{Liu} 2008)\end{array}$ & BH, LMXB & $\begin{array}{c}2.00 \mathrm{E}+21 \\
\text { (Bodaghee 2008) }\end{array}$ & $1.54 \mathrm{E}+36$ \\
\hline Cyg X-1 & $\begin{array}{c}5 d \\
(\text { Liu 2008) }\end{array}$ & $\begin{array}{c}2.14 \mathrm{kpc} \\
(\text { Liu 2008) }\end{array}$ & BH, HMXB & $\begin{array}{c}\text { 6.21E+21 } \\
\text { (Bodaghee 2008) }\end{array}$ & $8.80 \mathrm{E}+36$ \\
\hline Cyg X-3 & $\begin{array}{c}0.2 \mathrm{~d} \\
(\text { Liu } 2008)\end{array}$ & $\begin{array}{c}9 \mathrm{kpc} \\
\text { (Bodaghee 2008) }\end{array}$ & BH, HMXB & $\begin{array}{c}8.50 \mathrm{E}+22 \\
\text { (Bodaghee 2008) }\end{array}$ & $1.21 \mathrm{E}+38$ \\
\hline GX 304-1 & $\begin{array}{c}133 d ? \\
(\text { Liu 2008) }\end{array}$ & $\begin{array}{c}2.4 \mathrm{kpc} \\
(\mathrm{Liu} 2008)\end{array}$ & BH, HMXB & $\begin{array}{c}1.06 E+22 \\
\text { (Predehl 1995) }\end{array}$ & $8.93 E+34$ \\
\hline 1A $0535+262$ & $\begin{array}{c}111 d \\
(\text { Liu 2008) }\end{array}$ & $\begin{array}{l}2-2.9 \mathrm{kpc} \\
\text { (Liu 2008) }\end{array}$ & NS, HMXB & $\begin{array}{c}\text { 6.50E+21 } \\
\text { (Bodaghee 2008) }\end{array}$ & $1.27 \mathrm{E}+35$ \\
\hline Cen X-3 & $\begin{array}{c}2.09 \mathrm{~d} \\
(\text { Liu 2008) }\end{array}$ & $\begin{array}{c}8-10 \mathrm{kpc} \\
\text { (Liu 2008) }\end{array}$ & NS, HMXB & $\begin{array}{c}1.95 \mathrm{E}+22 \\
\text { (Bodaghee 2008) }\end{array}$ & $2.12 \mathrm{E}+37$ \\
\hline
\end{tabular}

TABLE I. XRBs from RXTE ASM data. The twenty-nine sources are listed along with orbital period, distance, classification, and hydrogen column density values found in literature searches. The calculated average x-ray luminosity is also included. 
Low- and Intermediate-Mass X-Ray Binaries

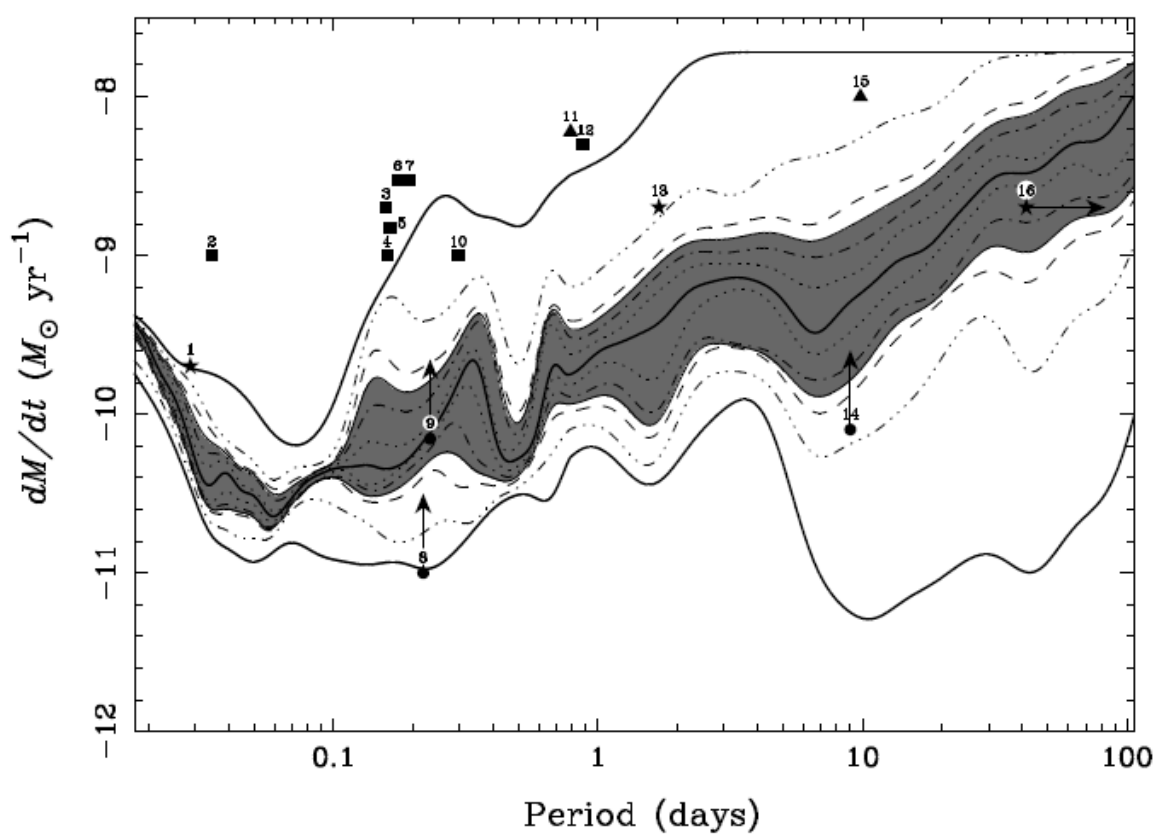

FIG. 18.- Cumulative (smoothed) probability distribution for the mass-accretion rate onto the neutron-star primary as a function of orbital period for the 100 binary calculations (with equal weighting for all sequences). The thick, solid central curve gives the median mass-accretion rate; the pairs of curves moving progressively outwards from the median curve include $20,40,60,80$, and 98 per cent of the distribution, respectively. The shaded region contains 50 per cent of all systems around the median. The symbols indicate the mass-transfer rates of selected observed X-ray binaries (triangles: Z sources; squares: atoll sources; stars: X-ray pulsars; circles: systems with accretion-disk coronae (lower limits]). The individual systems are (in ascending order of orbital period, as given in parentheses): (1) 1626-67 (0.69 hr), (2) 1916$053(0.83 \mathrm{hr})$, (3) $1636-536(3.8 \mathrm{hr})$, (4) 0748-676 (3.82 hr), (5) 1254-690 (3.93 hr), (6) GX9+9 (4.2 hr), (7) $1735-555$ (4.65 hr), (8) 2129+470 $(5.24 \mathrm{hr}),(9) 1822-37(5.57 \mathrm{hr}),(10) 1658-29(7.11 \mathrm{hr})$, (11) Sco X-1 $(18.9 \mathrm{hr}),(12), 1624-590$ (21 hr), (13) Her X-1 (40.8 hr), (14) 0921-630 $(216 \mathrm{hr}),(15)$ Cyg X-2 (236 hr), (16) GX1+4 (> $1000 \mathrm{hr})$ (taken from the catalogs by van Paradijs 1995 and Christian \& Swank 1997).

FIG. 3. Mass transfer rate versus period plot taken from the Podsiadlowski et. al. 2001 paper. The figure description from the paper has been retained.

much higher luminosities. It is interesting to note that if the black hole LMXB data was plotted together with the neutron star LMXB data, the black hole LMXB sources fell in to clusters with the neutron star LMXB sources, indicating that the type of compact body, be it a neutron star or black hole, has no influence on the periodluminosity relation.

The plot of the HMXBs (figure 2.3), which displayed the remaining five sources, displayed results that ran somewhat contrary to the LMXB data. The HMXBs clearly tended to decrease in luminosity as the period increased. There were no prior expectations for the HMXB data; however this does clearly show that the relation between luminosity and period behaves differently between HMXBs and LMXBs. It should be noted that all the HMXBs were plotted together, regardless as to whether the compact object was a neutron star or black hole, due to a shortage of data. Much like the LMXB data though, the classification of the compact body does not seem to have any relevance to the period-luminosity relation.

\section{CONCLUSION}

In conclusion, there needs to be further investigation into the relationship between period and luminosity for XRBs. While there seems to be a definite relation for HMXBs, the case is much different for LMXBs. The vast discrepancies in luminosity despite similar orbital periods for some LMXBs suggest the period-luminosity relation may not be as straight forward as previously thought. One avenue for further investigation could include determining more details, beyond mass of donor star and classification of the compact body companion, of some of the LMXBs as it may be the case of their being more factors at play in a period-luminosity.

In short, more research is required to yield more definite results regarding the relationship between $x$-ray luminosity and period, however preliminary results do look promising. I believe it is likely that the work done on this project will lead to results regarding a periodluminosity relation for XRBs in the near future, or if nothing else to further our current understanding of XRBs. 


\section{ACKNOWLEDGMENTS}

The quick-look results were provided by the ASM/RXTE team at MIT and the Goddard Space Flight Center Science Operations Facility and Guest Observer Facility. Thanks to Dr. Craig Heinke for his support and supervision while I worked on this project.

\section{REFERENCES}

[1-3,5] B.W. Carroll, D.A. Ostlie. An Introduction to Modern Astrophysics. Pearson, 2007.
[4] P. Podsiadlowski, S Rappaport, E. Pfahl. Evolutionary Binary Sequences for Low- and Intermediate-Mass X-ray Binaries. arXiv:astro-ph/0107261v1 (December 2, 2012).

[6] M. Tremmel et.al. Modeling the Redshift Evolution of the Norma Galaxy X-ray Luminosity Function. arXiv:1210.7185v2 [astro-ph.CO]. (December 2, 2012).

[7] http://heasarc.gsfc.nasa.gov/docs/xte/xte2.html. (December 2, 2012).

[8] http://heasarc.gsfc.nasa.gov/docs/xte/ASM.html. (December 2, 2012).

[9] http://heasarc.gsfc.nasa.gov/docs/xte/XTE.html. (December 2, 2012). 